\title{
Symmetrized exponential oscillator
}

\author{
Miloslav Znojil \\ Nuclear Physics Institute of the CAS, Hlavní 130, 25068 Řež, Czech Republic \\ and \\ Institute of Systems Science, Durban University of Technology, 4000 Durban, South Africa \\ e-mail: znojil@ujf.cas.cz
}

\section{Keywords:}

quantum bound states;

exactly solvable models;

Bessel special functions;

transcendental secular equation;

numerical precision;

\section{PACS numbers:}

PACS 03.65.Ge Solutions of wave equations: bound states

PACS 02.30.Gp Special functions

\begin{abstract}
Several properties of bound states in potential $V(x)=g^{2} \exp (|x|)$ are studied. Firstly, with the emphasis on the reliability of our arbitrary-precision construction, wave functions are considered in the two alternative (viz., asymptotically decreasing or regular) exact Bessel-function forms which obey the asymptotic or matching conditions, respectively. The merits of the resulting complementary transcendental secular equation approaches are compared and their applicability is discussed.
\end{abstract}




\section{Introduction}

One-dimensional bound-state Schrödinger equation

$$
-\frac{\mathrm{d}^{2}}{\mathrm{~d} x^{2}} \psi_{n}(x)+V(x) \psi_{n}(x)=E_{n} \psi_{n}(x), \quad \psi_{n}(x) \in L^{2}(\mathbb{R}), \quad n=0,1, \ldots
$$

with the centrally symmetric confining potential

$$
V(x)=g^{2} \exp (|x|), \quad x \in(-\infty, \infty)
$$

(cf. Fig. 1) does not seem to have attracted attention of the authors of textbooks on quantum mechanics. In the context of quantum phenomenology, such a neglect is certainly undeserved since the asymptotic growth of the potential may be perceived as positioned somewhere in between quadratic harmonic-oscillator $V^{(H O)}(x)=(\omega x)^{2}$ with equidistant, vibration-type spectrum of energies $E_{n}=(2 n+1) \omega, n=0,1, \ldots$ and its equally popular infinite-power square-well partner $V^{(S W)}(x)=(\omega x)^{\infty}$ leading to the quadratic growth of $E_{n}=\pi^{2}(n+1)^{2} \omega^{2} / 4, n=0,1, \ldots$ resembling the rotational energy bands [1].

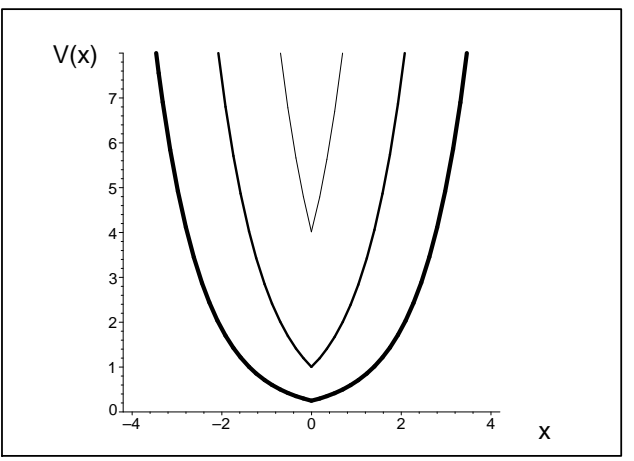

Figure 1: The shape of potential (2) at small $g^{2}=1 / 4$ (thick, almost smooth-looking curve), medium $g^{2}=1$ (medium curve) and larger $g^{2}=4$ (thin curve, sharply spiked shape).

The neglect of model (2) seems equally undeserved from the purely formal point of view because the related Schrödinger equation is extremely elementary and exactly solvable in terms of the modified Bessel functions matched in the origin [2, 3]. The low popularity of the conceptual as well as practical use of the bound-state families as described by Eqs. (11) and (2) may be given several tentative explanations. We shall return to this point later, in section 4 below. Now, let us only mention the feature of non-analyticity of the potential in the origin. Indeed, the analyticity along the whole real line is precisely what makes harmonic oscillators so popular, say, as toy models in quantum field theory [4] as well as in rigorous functional analysis [5, 6]. 
In what follows we intend to re-attract attention to the non-analytic model (11) + (2) and to describe and discuss a few basic properties of its exact solutions at some length, therefore. Let us also add that in a historical perspective our present interest in a deeper study of the "missing link" (2) connecting $V^{(H O)}(x)$ with $V^{(S W)}(x)$ found its origin in the methodical aspects of quantum gravity and, in particular, in the related Smilga's comment [7] on the dynamics of the so called Pais-Uhlenbeck toy-model oscillator. Indeed, in the latter model the dynamics is mimicked using a pair of potentials $V^{(H O)}(x)$ with different $\omega$ s (cf. Eqs. Nr. 5 and 6 in loc. cit.). In our most recent paper [8] we tried to test some of the Smilga's conclusions using an alternative, Pais-Uhlenbeck-like model in which the dynamics was generated by square-wells $V^{(S W)}(x)$ with different $\omega \mathrm{s}$ (cf. Eqs. Nr. 1 and 2 in loc. cit.). Unfortunately, the results of the comparison proved too model-dependent. In other words, the square-well simulation of the Pais-Uhlenbeck-related effects were, from the point of view of the original problem of quantum gravity, next to useless. Thus, the study of the present model (2) can be perceived as offering a new hope and having a rather deep motivation, especially due to its exact solvability.

\section{Mathematical aspects of the model}

\subsection{Exact solvability}

On the Wolfram's webpage [2] one easily finds that differential Eq. (11) + (2) re-written in the form

$$
-y^{\prime \prime}(x)+a \exp (b x) y(x)=c y(x)
$$

has general solution

$$
\begin{gathered}
y(x)=k_{1} e^{\pi \sqrt{c} / b} \Gamma(1-2 i \sqrt{c} / b) I_{-2 i \sqrt{c} / b}\left(2 \sqrt{a e^{b x}} / b\right)+ \\
+k_{2} e^{-\pi \sqrt{c} / b} \Gamma(1+2 i \sqrt{c} / b) I_{2 i \sqrt{c} / b}\left(2 \sqrt{a e^{b x}} / b\right)
\end{gathered}
$$

where $I_{n}(z)$ denotes the modified Bessel function of the first kind and where $\Gamma(x)$ is the gamma function.

It is well (and for a long time, [9]) known that the existence of the latter closed-form solutions renders the construction of the states which are generated by the present confining interaction (2) non-numerical (or semi-numerical at worst, cf. [3]). What is much less often acknowledged is that the special-function solvability of the problem opens also the way towards various forms of its innovative deformations. Let us recall here just two illustrative examples. In the first one one can require a complete survival of the quantum stability of the system in question (i.e., of the strict reality of the energy spectrum) even after the real potential gets replaced by its suitable 
complexified form. In Ref. [10], for example, the authors achieved such a goal via an elementary replacement $b \rightarrow \mathrm{i} b$ in Eq. (3) (cf. loc. cit. for more details).

In the role of the second illustrative example of the change of horizons based on the analytic continuation techniques we may recall the change of parameters $(a, b) \rightarrow(-a,-b)$ in Eq. (3) which is of interest in molecular physics. In such an alternative dynamical regime one studies, typically, the resonances (i.e., complex eigenvalues) which are generated by a "realistic", asymptotically vanishing real potential with a repulsive core in the origin (cf., e.g., Ref. [11] and references therein).

In what follows our attention will remain restricted to the most straightforward bound-state interpretation (11) + (2) of the exponential interaction and of the Bessel's form of differential Eq. (3).

\subsection{Consistency of the non-analyticity at $x=0$}

In the spirit of our recent brief comment [12] on ordinary differential Schrödinger equations let us first remind the readers that the existence of the single and isolated point of non-analyticity of any given potential $V(x)$ in the origin may be given a very natural interpretation in the context of the theory of quantum graphs. In this language, any one-dimensional Schrödinger equation may be identified with a quantum graph with one vertex and two edges. In practice this means that the equation is split into a pair of half-line differential equations

$$
\begin{aligned}
& -\frac{\mathrm{d}^{2}}{\mathrm{~d} s^{2}} \psi_{(l e f t)}(s, E)+g^{2} e^{-s} \psi_{(l e f t)}(s, E)=E \psi_{(l e f t)}(s, E), \quad s \in(-\infty, 0), \\
& -\frac{\mathrm{d}^{2}}{\mathrm{~d} r^{2}} \psi_{(\text {right })}(r, E)+g^{2} e^{r} \psi_{(\text {right })}(r, E)=E \psi_{(\text {right })}(r, E), \quad r \in(0, \infty)
\end{aligned}
$$

such that the logarithmic derivatives of the two respective halves of the wave function are properly matched in the origin,

$$
\psi_{(l e f t)}(0, E)=\psi_{(\text {right })}(0, E), \quad \psi_{(l e f t)}^{\prime}(0, E)=\psi_{(\text {right })}^{\prime}(0, E) .
$$

Such a split of $\psi_{n}(x)$ into two halves reflects the spatial symmetry of the interaction, $V(x)=V(-x)$ so that we are allowed to restrict attention, say, just to the right half-axis (so we may drop the subscript "right" as redundant), distinguishing just between the conventionally normalized evenparity bound states such that

$$
\psi_{(\text {even })}(0, E)=1, \quad \psi_{(\text {even })}^{\prime}(0, E)=0, \quad E=E_{n}, \quad n=0,2,4, \ldots
$$

and the odd-parity bound states such that

$$
\psi_{(\text {odd })}(0, E)=0, \quad \psi_{(\text {odd })}^{\prime}(0, E)=1, \quad E=E_{n}, \quad n=1,3,5, \ldots
$$


Let us add that the left-right split of the problem may be also perceived as a one-dimensional parallel to the partial-wave expansion of the wave functions in three dimensions. In such a perspective one of Eqs. (4) or (5) plays the role of an analogue of the radial Schrödinger equation. Similarly, the operator of parity $\mathcal{P}$ may be perceived as a one-dimensional counterpart of the rotational symmetry of the three-dimensional central $V(\vec{x})=V(|\vec{x}|)$.

In the same spirit, the three-dimensional quantum number of angular momentum $\ell=0,1, \ldots$ degenerates here to the two values of the parity quantum number \pm 1 . Thus, we may conclude that the non-analyticity of our present potential (2) in the origin finds its analogue in the optional central singularities exhibited, e.g., by the Coulombic attraction $V(|\vec{x}|) \sim-1 /|\vec{x}|$ or by the more singular repulsive cores (e.g., $V(|\vec{x}|) \sim|\vec{x}|^{-6}[13]$ ) in three dimensions.

\subsection{Asymptotically decreasing solutions and the exact energies}

Via the change of independent variable $r \rightarrow y=\exp (r / 2)$ one transforms the radial-like differential Eq. (5) (living, at positive energies $E>0$, on the half-line of coordinates $r=|x| \in(0, \infty)$ ) into another differential equation which is solvable in terms of Hankel functions (alias Bessel functions of the third kind - see their definition in [14]).

At an arbitrary trial energy $E=k^{2}$ this yields all of the admissible general solutions of Eq. (5) in the compact, exact and explicit special-function form

$$
\psi_{(\text {general })}\left(r, k^{2}\right)=C_{1} H_{\nu}^{(1)}(z)+C_{2} H_{\nu}^{(2)}(z), \quad z=2 i g e^{r / 2}, \quad \nu=2 i k .
$$

Once we require, in the bound-state context, the asympotically decreasing behavior of these solutions we may consult formulae 10.2.5 and 10.2.6 in Ref. [14] and conclude that we must put $C_{2}=0$. The resulting, asymptotically correct solutions have the compact and final first-Hankelfunction form

$$
\psi_{(\text {asymptotically decreasing })}\left(r, k^{2}\right) \sim H_{2 i k}^{(1)}\left(2 i g e^{r / 2}\right) .
$$

Naturally, this solution varies with the trial-energy variable $E=k^{2}$ but the value of this variable must be fixed via the transcendental secular equations (7) (even-parity bound-state energies) or (8) (odd-parity bound-state energies), i.e., via the respective transcendental-equation constraint at $r=0$, viz.,

$$
\frac{d}{d g} H_{2 i k}^{(1)}(2 i g)=0, \quad n=0,2,4, \ldots
$$

or

$$
H_{2 i k}^{(1)}(2 i g)=0, \quad n=1,3,5, \ldots
$$


These equations must be solved numerically of course. The former, slightly more complicated one may be still given the two alternative but equivalent simplified forms, viz.,

$$
H_{2 i k-1}^{(1)}(2 i g)-\frac{g}{k} H_{2 i k}^{(1)}(2 i g)=0, \quad n=0,2,4, \ldots
$$

or

$$
H_{2 i k-1}^{(1)}(2 i g)-H_{2 i k+1}^{(1)}(2 i g)=0, \quad n=0,2,4, \ldots
$$

Both of these simplifications follow from formula 10.6. 1 in [14]. The construction is complete.

\section{Physical aspects of the model}

Although the detailed and exhaustive analysis of the precision of the numerical determination of the bound-state energies $E=k^{2}$ via secular equations (11) - (14) lies far beyond the scope of the present letter, several features of these equations deserve an explicit commentary.

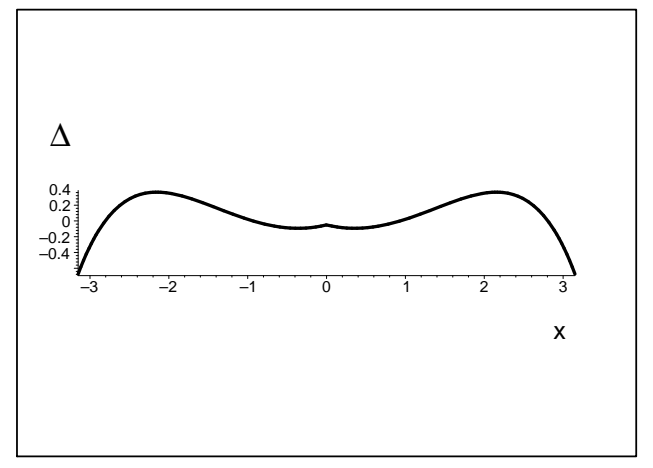

Figure 2: The short-range smallness of difference between harmonic oscillator and exponential potential (2) $\left(\Delta=0.5 \cdot x^{2}+0.2-g^{2} \cdot \exp |x|\right.$ with $\left.g^{2}=1 / 4\right)$.

\subsection{The loss of precision in the domain of very small couplings $g$}

From our illustrative Fig. 1 (and, in particular, from its complement Fig. 2) one could deduce that in the dynamical regime of very small couplings $g$ the shape of our potential (2) is not too different from the shape of harmonic oscillator, within the range of the low-lying spectrum at least. Hence, the exponential-well spectrum could also, in some sense, resemble the spectrum of the harmonic oscillator. Naturally, this does not imply that its search must necessarily be also numerically well behaved. A word of warning may be found, e.g., in Ref. [11] in which it has been argued that in the numerical practice and, in particular, in the case of exponential potentials it is often useful 
to test the precision of the numerical root-searching results using an alternative algorithm (in particular, in loc. cit. the authors recommended the use of the so called Riccati-Padé method).

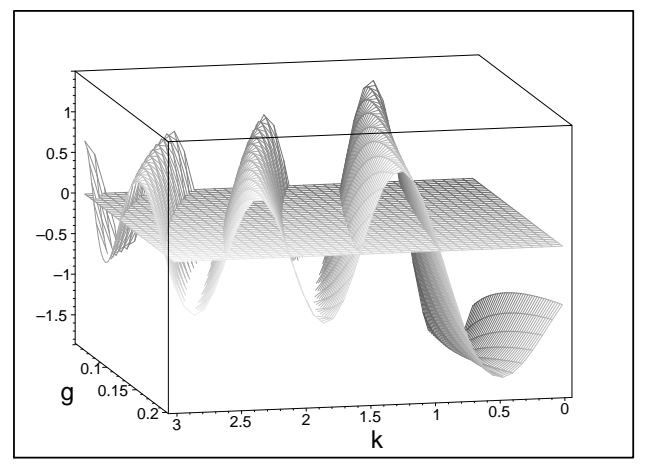

Figure 3: The sample of the shape of the asymptotically decreasing solution (10) at the small couplings $g$ (its intersections with the horizontal plane define the physical energies $E_{n}=k_{n}^{2}(g)$ as functions of $g$ at $n=1,3,5, \ldots)$.

Via an explicit numerical test paying attention just to the simplest secular Eq. (12) in the odd-parity case, a definite encouragement of our present optimism was provided by the picture showing the smooth parameter-dependence of the asymptotically decreasing solutions (see Fig. 3). Moreover, the real-function nature of the picture illustrates that there exists a suitable $a d$ hoc normalization which keeps our asymptotically decreasing function of variables $g$ and $k$ real.

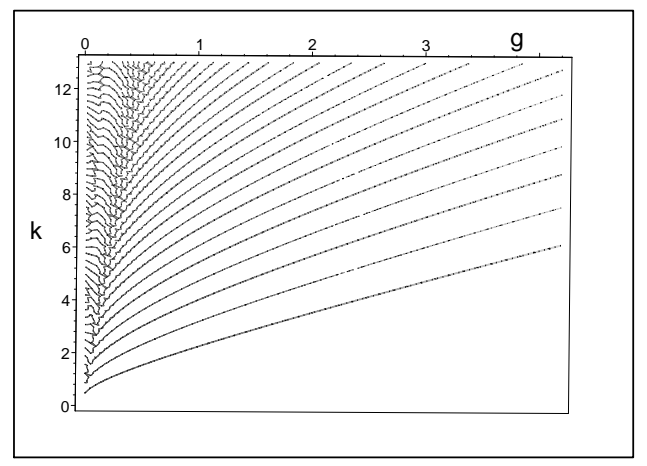

Figure 4: The numerically determined odd-parity zeros $k=k_{n}(g), n=1,3,5, \ldots$ of the asymptotically-decreasing-solution secular equation (12).

The latter two observations (plus their parallels in the even-parity case) explain the straightforward nature of the algorithms of the numerical search for the bound-state roots of the exponentialwell secular equations. For this reason we were able to extend the computations based on the 
asymptotically-decreasing-solution algorithms beyond the small-coupling domain. The results are sampled in Fig. 4 .

\subsection{Regular solutions and the both-sided estimates of the energies}

A more detailed inspection of the numerical results as presented in Fig. 4 reveals that the use of the fixed-precision arithmetics leads to the loss of the reliability of the localization of the boundstate roots $k_{n}(g)$ in the domain of small $g<g_{\text {critical }}(n)$. Empirically we see a more or less linear $n$-dependence of $g_{\text {critical }}(n)$, with maximal $g_{\text {critical }}(45) \approx 0.57$ at the highest level as identified in Fig. 4.

Naturally, such a loss of precision is not too surprising. In fact, our a priori decision of preferring the asymptotically decreasing solutions (10) proved rather lucky because Fig. 4 appeared to provide a reliable information about the spectrum in the more difficult and non-perturbative "strong-coupling" dynamical domain.

This being said, the exact solvability of our radial-like Schrödinger equation still admits the use of an alternative strategy in which one would start from the initial conditions (7) and (8) and in which one would construct the so called regular solutions $\psi_{(\text {regular })}\left(r, k^{2}\right)$ by the purely analytic and non-numerical means. In such a setting, naturally, the physical values of $k=\sqrt{E_{n}}$ will have to be sought via the fit of the regular solutions to the standard (i.e., Dirichlet) asymptotic boundary conditions,

$$
\psi_{\text {(regular })}\left(R, k^{2}\right)=0, \quad R \gg 1 .
$$

One can expect that such an alternative construction strategy could cover not only the domains of parameters in which the above-described asymptotically-decreasing-solution approach had failed but also some of the applications of the solutions in which one needs a very precise evaluation of the wave functions.

Although the regular-solution algorithm could start, in principle, from the same change of variables as above, our analysis showed that the analytic formulae putting emphasis on the matching at $r=0$ become simpler if we change some signs and use the abbreviations

$$
\begin{gathered}
K_{\nu}(z)=\frac{i \pi}{2} \exp \left(\frac{i \pi}{2} \nu\right) H_{\nu}^{(1)}(i z) \\
I_{\nu}(z)=\exp \left(-\frac{i \pi}{2} \nu\right) J_{\nu}\left(\exp \left(\frac{i \pi}{2}\right) z\right) .
\end{gathered}
$$

In this manner, the modified, small-r-friendly ansatz

$$
\psi_{(\text {regular })}\left(r, k^{2}\right)=D_{1}(\nu, g) K_{\nu}(w)+D_{2}(\nu, g) I_{\nu}(w), \quad w=w(g, r)=2 g e^{r / 2}, \quad \nu=2 i k
$$


leads to the parity-dependent final results again. Thus, up to a modifiable overall normalization the choice of the even parity may be shown to yield, after the straightforward though still rather tedious computations, the coefficients given by the derivative-related formulae

$$
D_{1}(\nu, g)=g I_{\nu+1}(2 g)+i k I_{\nu}(2 g), \quad D_{2}(\nu, g)=g K_{\nu+1}(2 g)-i k K_{\nu}(2 g) \quad \text { (even parity) }
$$

The choice of the odd parity leads to simpler expressions,

$$
D_{1}(\nu, g)=-I_{\nu}(2 g), \quad D_{2}(\nu, g)=K_{\nu}(2 g) \quad(\text { odd }- \text { parity case })
$$

Having these formulae at our disposal we are now prepared to test the numerical performance of the regular-solution-based recipe.

At any suitable trial energy variable $E=k^{2}$ the recipe requires that we formulate the secular equation for the physical bound-state energies as the Dirichlet asymptotic boundary condition. In the numerical practice this means that we preselect a suitable, sufficiently large value $R$ of the coordinate and, in both the even- and odd-parity cases, search for all of the roots $k=k_{n}(R)$ of transcendental Eq. (15). In the limit of large $R$ this form of secular equation should again determine the exact physical values of the energy levels $E=E_{n}=k_{n}^{2}(\infty)$.

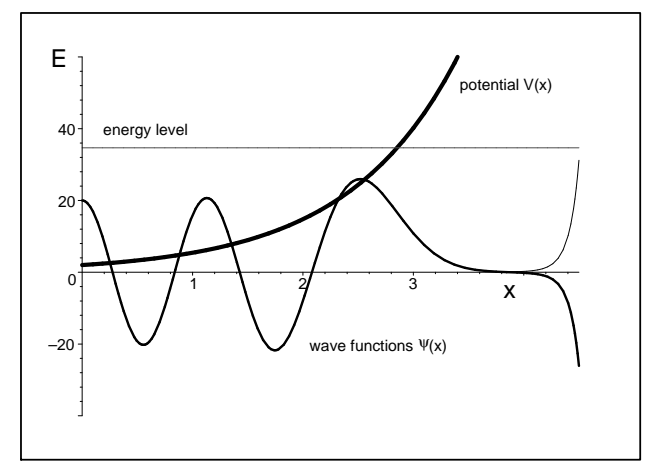

Figure 5: The sample of $V(x)$ (at $g^{2}=2$ ), of one of its highly-excited even-parity bound-state energy (viz., of $E_{n}$ with $n=8$ ) and of the related upper- and lower-bound radial-like wave-function $\psi_{n}(r)$, with normalization at $r=0$ being re-scaled here ad hoc.

As we already indicated the key appeal of the use of regular solutions (18) lies, a priori, in a more friendly representation of the wave functions. This expectation is confirmed by Fig. 5 in which the visible difference between the wave functions evaluated at the minorizing and majorizing $k=k_{\text {physical }} \pm 0.0001$ only becomes detectable beyond $r \approx 4$. In parallel, Table 1 shows that the sufficient cut-off $R$ need not lie too far beyond the intersection $x_{0}$ of the energy level with the exponentially growing wall of the potential well. 
Table 1: The sample of parameters for the numerical bracketing of the even-parity energies using secular Eq. (15) $\left(\psi(R)=0, E=V\left(x_{0}\right)\right)$.

\begin{tabular}{||c||c|c|c|c||}
\hline \hline $\mathrm{n}$ & $E_{n}^{\text {(lower bound })}$ & $E_{n}^{(\text {upper bound })}$ & $R$ & $x_{0}$ \\
\hline \hline 0 & 4.12005 & 4.12010 & 3.0 & 0.72 \\
2 & 11.0065 & 11.0075 & 3.0 & 1.71 \\
4 & 18.2822 & 18.2830 & 3.4 & 2.21 \\
\hline \hline
\end{tabular}

\section{Discussion}

In the present note we demonstrated that the elementary transition from the full-line equation (11) to the half-line equation (5) enables one to claim that in spite of the admitted non-analyticity in the origin our present model can be perceived as exactly solvable. In other words, we believe that the conventional families of the exactly solvable one-dimensional potentials (with their typical list provided by review paper [15]) should be complemented by the symmetric functions $V(x)=V(-x)$ which are non-analytic in the origin but still tractable via special functions (cf. also several other comments [3, 16, 17, 18, 19, 20, 21] in this respect).

Once more, let us remind the readers that our choice and study of potential (2) was motivated by the need of interpolation between the ubiquitous harmonic oscillator $V^{(H O)}(x)=\omega^{2} x^{2}$ and its equally easily solvable square-well alternative

$$
V^{(S W)}(x)= \begin{cases}\infty, & x<-L \\ 0, & x \in(-L, L) \\ \infty, & x>L\end{cases}
$$

with, say, $L=1 / \omega$. Such a need of interpolation may have various pragmatic as well as theoretical reasons [22]. Nevertheless, one of the main difficulties encountered during such a search is usually seen in an inadvertent loss of the appealing, textbook-explained exact solvability (ES) status of the underlying one-dimensional bound-state Schrödinger Eq. (11).

In practice, due to the ordinary linear differential nature of Eq. (11) it is still fortunate that one can employ standard numerical methods and one can construct the bound state solutions, with arbitrarily predetermined precision, for a vast majority of the potentials of phenomenological interest. Still, one usually argues that the strictly non-numerical nature of bound states makes the ES interactions $V^{(H O)}(x)$ and $V^{(S W)}(x)$, in many a respect, privileged.

By our opinion, the key weakness of the latter argument lies in the vagueness of the very definition of the ES status. This definition proves even different for the smooth, analytic potentials and for the various discontinuous versions and descendants of square wells for which the wave 
functions remain elementary but for which, in a way illustrated, e.g., in Refs. [23, 24, 25, 26], the energies must still be sought - purely numerically - as roots of transcendental equations. In the former case, on the contrary, the energies are usually given by closed and elementary formulae while the wave functions themselves remain elementary only due to a slightly mysterious degeneracy of the infinite-Taylor-series special-function general solutions to classical orthogonal polynomials (cf., e.g., [15] for a representative list of the analytic ES interaction models as sampled here by $\left.V^{(H O)}(x)\right)$.

As a true test of the robustness of the ES concept we studied here the "non-ES" toy-model interaction (2) which can be also considered, cum grano salis, solvable. In addition, potential (2) shares certain geometrical as well as solvability features with both of the above-mentioned ES examples. At the small couplings $g^{2}>0$ and in the low-lying energy region its shape resembles harmonic oscillator (cf. Fig. 2 where $g^{2}=1 / 4$ ). With the growth of the coupling the spike at $x=0$ becomes more pronounced (return to Fig. 1 where the value of $g^{2}$ varied from $1 / 4$ to 4 ).

It is obvious that with the growth of $g^{2}$ the spectrum is being pushed upwards. Moreover, at higher excitations the function (2) of $x$ becomes steeper so that the potential resembles, more and more, square well. In contrast to the equidistant harmonic-oscillator case the $n \gg 1$ differences $\delta_{n}=E_{n+1}-E_{n}$ between the neighboring levels may be, therefore, expected to grow - more or less quadratically - with $n$. Indeed, such an expectation is numerically confirmed by Fig. 6

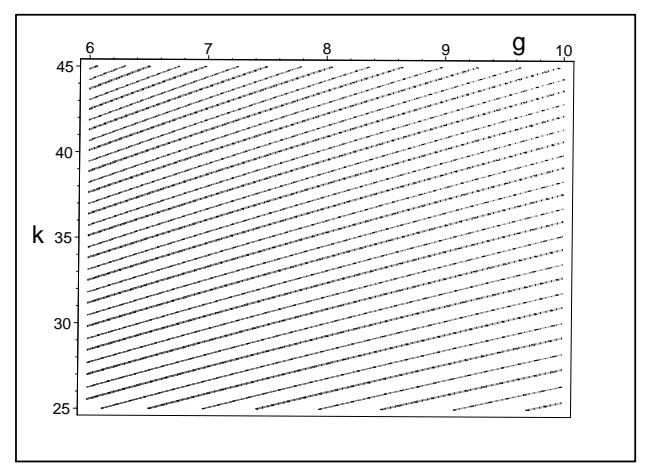

Figure 6: The sample of the approximate equidistance of the square roots $k_{n}=\sqrt{E_{n}}$ of the highly-excited even-parity bound-state energies in the domain of $g \in(6,10)$ and $k \in(25,40)$.

From the purely phenomenological point of view interaction (2) is made interesting by an interplay between the latter two features. The model seems also remarkable on the purely formal grounds because, as we showed, the underlying bound-state Schrödinger equation (11) proves piecewise exactly solvable in terms of the various forms of Bessel functions. Naturally, this could open a way towards various further generalizations. 
Last but not least we would like to emphasize that we also showed here how one can circumvent the usual precision-fighting difficulties. Incidentally, some of these difficulties are intimately connected with the extremely quick exponential growth of the walls of our potential well (2) at large distances. In this context we may also recall Ref. [27] in which, in the context of a simplified description of the deuteron, a similar necessity of the cross-checking verification of the numerical reliability of the exact $s$-wave formulae has been noticed to occur in the opposite extreme of the exponentially decreasing potentials.

\section{Acknowledgements}

The project was supported by IRP RVO61389005 and by GAČR Grant Nr. 16-22945S. 


\section{References}

[1] F. Constantinescu and E. Magyari, Problems in Quantum Mechanics. Pergamon, Oxford, 1976 , p. 36.

[2] http://www.wolframalpha.com/input/?i=-y $\% 27 \% 27(\mathrm{x}) \% 2 \mathrm{Baexp}(\mathrm{bx}) \mathrm{y}(\mathrm{x}) \% 3 \mathrm{Dcy}(\mathrm{x})$

[3] http://physics.stackexchange.com/questions/47128/eigenvalues-and-eigenfunctionsof-the-exponential-po

[4] J. D. Bjorken and S. D. Drell, Relativistic Quantum Fields. McGraw-Hill, New York, 1965.

[5] F. H. Szafraniec, Operators of the Quantum Harmonic Oscillator and its Relatives, in F. Bagarello, J. P. Gazeau, F. H. Szafraniec, and M. Znojil, editors, "Non-Selfadjoint Operators in Quantum Physics: Mathematical Aspects", John Wiley \& Sons, Hoboken, 2015, pp. 59 120.

[6] F. Bagarello, Deformed Canonical (Anti)Commutation Relations and Non-self-adjoint Hamiltoians, in F. Bagarello, J. P. Gazeau, F. H. Szafraniec, and M. Znojil, editors, "Non-Selfadjoint Operators in Quantum Physics: Mathematical Aspects", John Wiley \& Sons, Hoboken, 2015, pp. $121-188$.

[7] A. Smilga, SIGMA 5 (2009) 017 (arXiv 0808.0139).

[8] E. Pelantová, Š. Starosta and M. Znojil, J. Phys. A: Math. Theor. 49 (2016) 155201.

[9] S. T. Ma, Phys. Rev. 69 (1946) 668.

[10] F. Cannata, G. Junker and J. Trost, Phys. Lett. A 246 (1998) 219.

[11] P. Amore, F. M. Fernández, Phys. Lett. A 372 (2008) 3149.

[12] M. Znojil, Can. J. Phys. 90 (2012) 1287.

[13] M. Znojil, Phys. Lett. A 158 (1991) 436.

[14] F. W. J. Olver and L. C. Maximon, Bessel Functions, in NIST Digital Library of Mathematical Functions (Chapter 10). Open access at address http://dlmf.nist.gov/10

[15] F. Cooper, A. Khare and U. Sukhatme, Phys. Rep. 251 (1995) 267.

[16] R. Sasaki and M. Znojil, arXiv:1605.07310. 
[17] A. M. Ishkhanyan, EPL 112 (2015) 10006.

[18] M. Znojil, Phys. Lett. A 381 (2016) 1414.

[19] M. Znojil, Mod. Phys. Lett. A 31 (2016) 1650088.

[20] C. Quesne, Quasi-exactly solvable symmetrized quartic and sextic polynomial oscillators, submitted

[21] A. V. Turbiner, arXiv:1603.02992 (Phys. Reports, in print).

[22] U. Guenther, F. Stefani and M. Znojil, J. Math. Phys. 46 (2005) 063504.

[23] M. Znojil, Phys. Lett. A 285 (2001) 7.

[24] M. Znojil and G. Lévai, Mod. Phys. Lett. A 16 (2001) 2273.

[25] C. Quesne, B. Bagchi, S. Mallik, H. Bila, V. Jakubsky and M. Znojil, Czech. J. Phys. 55 (2005) 1161.

[26] M. Znojil, J. Phys. A: Math. Gen. 39 (2006) 441.

[27] S. Flügge, Practical Quantum Mechanics I. Springer, Berlin, 1971, pp. 196 - 200. 